\title{
Time-Mean Helicity Distribution in Turbulent Swirling Jets
}

Helicity offers an alternative approach to investigations of the structure of turbulent flows. Knowledge of the spatial distribution of the time-mean component of helicity is the starting point. Yet very little is known even about basic cases in which Helicity plays important role, such as the case of a swirling jet. This is the subject of the present investigations, based mainly on numerical flowfield computations. The region of significantly large time-mean helicity density is found only in a rather small region reaching to several nozzle diameters downstream from the exit. The most important result is the similarity of the helicity density profiles.

Keywords: helicity, chirality, jets, swirling flows, swirl generator, supercirculation.

\section{Introduction}

The concept of helicity was originally introduced in the $1950 \mathrm{~s}$ in the context of magnetohydrodynamics, where it proved to be very useful and has been used quite extensively. Its use in hydromechanics of non-conducting fluids was introduced in 1969 by Moffat [1], who proposed to apply it as a measure of the "knottedness" of vortex lines - which are known to be often quite tangled in turbulent flows. Helicity is a quantity of very fundamental character, closely related to basic topological aspects of the flowfield. Moreau [4] formulated the helicity conservation theorem. This holds for ideal non-viscous fluids, in particular for any inviscid barotropic fluid flowing under the influence of conservative body forces. In the theory of non-viscous flows, this invariance gives helicity a status comparable to that of quantities as basic as energy. There have been several attempts to use helicity also as the starting point in attacking the problem of fluid flow turbulence. Recently, interest and hopes have revived, as documented e.g. in [5, 6], and [8] - and particularly in the studies by Zimmerman [11, 12, 13] of helical coherent structures in turbulence, which have led to important results having direct practical engineering relevance. The present study of helicity distribution in a swirling jet was performed to support a more extensive current research program aimed at applying the concept to description and improvement of fuel and oxidant mixing in swirl burners.

The helicity of fluid flow [2] is defined as an integral (across a specified volume) of the scalar product of local velocity vector $w$, characterising the velocity field, and local vorticity vector $\omega$, which characterises the rotational motions - as shown in Figs. 1 and 2. In practical flowfield studies, the variable of interest is the local quantity, helicity density $\mathcal{H}$ $\left(\mathrm{m} / \mathrm{s}^{2}\right)$ as defined in Fig. 2:

$$
\begin{aligned}
\mathcal{H} & =w \omega \\
& =w|\omega| \cos \alpha \\
& =w_{1} \omega_{1}+w_{2} \omega_{2}+w_{3} \omega_{3} \ldots
\end{aligned}
$$

An interesting fact is that despite being a quantity fully determined by a single numerical value, helicity is not a simple scalar. Its sign depends upon the used reference frame. Such quantities are sometimes characterised as pseudo-scalars: the change of sign takes place during the transition from the reference frame of right-handed chirality to left-handed one.

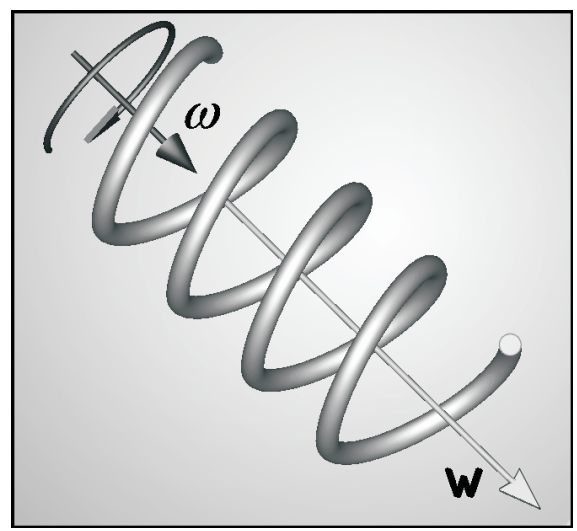

Fig. 1: Helical deformation of a fluid volume in turbulent flows. This idealised shape would arise in the exceptional case of strong rotation with mutually collinear vorticity and velocity vectors.

The idea of the helical shape of fluid objects deformed by a flowfield exhibiting high helicity is presented in Fig. 1. It is a very idealised case, with the two vectors $w$ and $\omega$ collinear, the rotational speed very high, and the deformation very regular. Examples of such shapes are known e.g. from tornadoes. Nevertheless in usual turbulent flows, especially with generally inclined local vectors $w$ and $\omega$ (Fig. 2) and irregular deformed objects, the helical shape is much less easy to recognise.

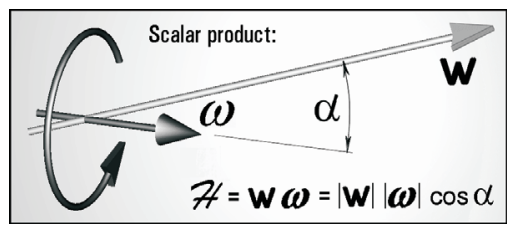

Fig. 2: The quantity evaluated in the present investigation is the helicity density $\mathcal{H}$ - the scalar product of the local velocity vector $w$ and vorticity vector $\omega$.

The deformation affects any object in the fluid. Objects of particular interest are turbulent vortices. A vortex, visualised by e.g. dye addition, exposed to a shear flow is elongated and twisted as if it were a deformable body, because vortices cannot be simply destroyed (they decay only gradually - in an ideal frictionless fluid their vorticity $\omega$ is conserved) and cannot be torn into pieces - this is prevented the consequences of Kelvin's theorem, the essence of which [3] may be stated as vortex cores behaving like material objects retaining their 
identity. Once the vortex core is bent, it becomes twisted and curled, even if it were alone in the flowfield, by the induced rotational motion generated by interaction with its other parts. Much more tangled shapes due to the induced motions are then caused by presence of other vortices in the neighbourhood. Due to the stochastic nature of the motions in typical turbulent flows the deformation is irregular. As a result, the real shapes of the visualised coherent vortical structures generally bear only a very distant resemblance to the regular shape indicated in Fig. 1. The "pitch" between the loops is usually large relative to the diameter of the object, and the stochastic character of the deformation causes the "pitch magnitude" to be different at different locations. The shapes are also typically skewed due mutual inclination of the two vectors (Fig. 2), which again may vary from one location to another. Finally, the lifetime of vortical structures is usually too short to permit seeing more than a single loop. Nevertheless the idea of the real deformation being composed from superimposed components corresponding to Fig. 1 is a useful working concept. In fact, the shapes of the deformed vortices need not be helical at all, and they may exhibit nonzero helicity. Moffat in [1] has evaluated the helicity of the interlooped configuration of vortices as shown in Fig. 3, and has demonstrated it to be proportional to the product of their circulations $\Gamma$ and a topological invariant called the linking number of the two loops. Configurations similar to those in Fig. 3, typically with several smaller loops instead of just the single one shown here, arise in the process of mutual interaction of structures, once the intertwined loops start to undergo the processes of "cut and connect" - a mechanism which may be particularly relevant for evaluating the spectral transfer of helicity.

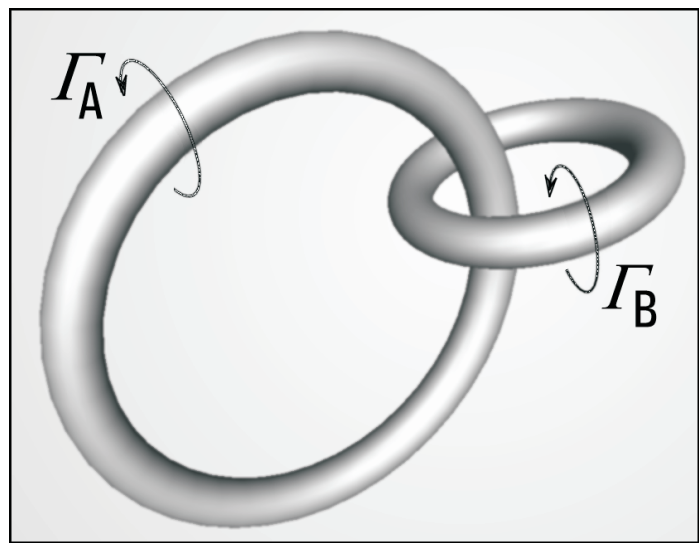

Fig. 3: A high helicity in a fluid flow does not necessarily mean the presence the of helical coherent structures of Fig. 1. Much more important may be the high helicity found for the mutually linked vortex rings (which, according to some theories, may be the basic structures in turbulence).

The starting point in investigations of helicity transport phenomena is the spatial distributions of time-mean helicity on the integral (or device) scales. Unfortunately, very little information is available, despite the engineering importance of many flows with a particularly strong helical spinning mode (lacking axial symmetry). The swirling character may be imparted externally. However, swirling flows, like the well known case of the bathtub vortex, typically exhibit self-induced rotation as a result of breaking the initially helicity-free reflectional symmetry.
The purpose of this paper is to provide some information about recent investigations of time-mean helicity in a very basic flow case - that of a round jet with rotation about its axis, caused by imparting a swirl upstream inside the nozzle. Both experimental, using PIV, and also computational studies were performed. So far most of the results have been obtained from the computations. The experiments have been delayed by the necessity to amend the standard optical interrogation system by additional components which generate a parallel different colour light sheet to resolve the derivatives in evaluating the vorticity. The resultant unique PIV system is specially suitable for helicity measurements. In the computations, helicity has been evaluated at individual points of the flowfield by multiplying the local collinear components of velocity and vorticity and summing of the three products, Eq. (1).

\section{Novel swirl generator}

Nozzles for generating swirling jets are commonly based upon one of the two usual types of swirl generators. On the one hand, there are passive devices inserted into the nozzle (or immediately upstream), the geometry of which disrupts the geometric axial symmetry. The most common forms are twisted tapes, helical rifling of the nozzle walls, or inserted inclined vanes. This method of generating the swirling motion was unsuitable for the experimental part of the present project, as it does not allow variations in the intensity of the generated swirl - unless the swirl imparting vanes are made with variable inclination, as discussed below. On the other hand, there are active devices, with the swirl generated by admission of an adjustable tangential inflow component superimposed

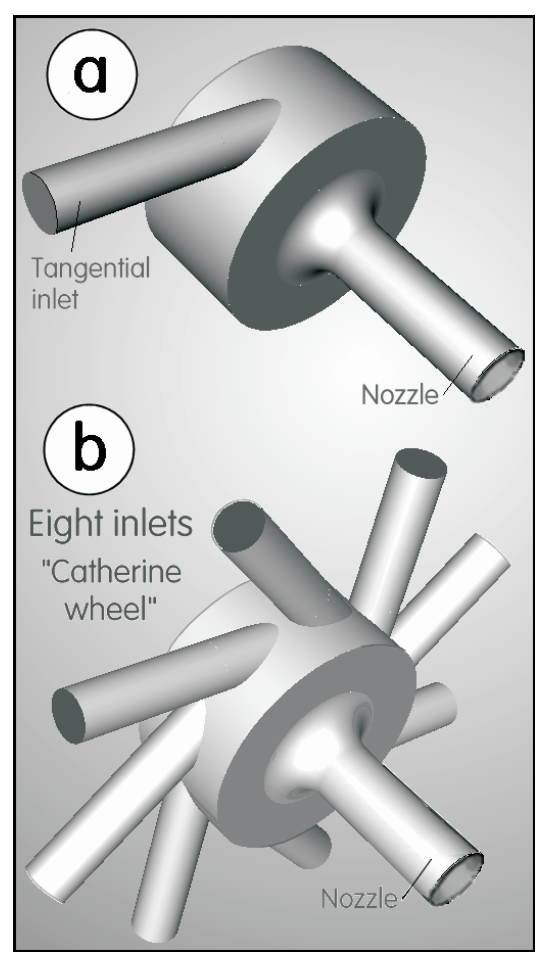

Fig. 4: Tangential inlet generation of swirling flows which was found unsuitable for the experimental study: a) single inlet produces asymmetric flow and instability, b) complicated multi-inlet layout can eliminate the asymmetry but retains instability and uneven nozzle exit velocity profile. 
on the basic axial flow (which, in the extreme case, may even vanish). Experience with vortex type fluidic devices, that use this tangential inflow approach is not encouraging for application in basic hydrodynamic investigations. The simple single-inlet geometry (a in Fig. 4) is unable to generate a sufficiently symmetric flow. This may be improved by a more complex arrangement with many inlets (such as case b in Fig. 4), with the disadvantage of a rather complex and more expensive layout - the more so because in the present case the planned investigations call for ambi-chirality, capability to generate both directions (senses) of rotation. Another problem caused by imparting the tangential momentum on the periphery of the inlet is the difficulty of obtaining a regular velocity profile at the exit. The profile shape may be improved by a long nozzle exit channel, but this generates a thick boundary layer (degrading the outer parts of the profile) and the inevitable fast dissipation of the swirl decreases the achievable magnitude of nozzle exit helicity.

A configuration meeting the demands is found in generators with adjustable vanes - adjusted to a desirable attack angle. They impart the momentum across the whole radius so that the resultant profile is regular. Unfortunately they tend to be mechanically very complicated and therefore expensive - especially if the adjustment is to be reproducible, which requires the vanes to be provided with some mechanism making their angle of attack equal and fixed. Though perhaps possible in principle, there have been few (if any) attempts to make the mechanism capable of performing time-dependent variation of the attack angle.

Instead of mechanically adjustable vanes, the author used for imparting the angular momentum in the upstream enlarged inlet cross section of the nozzle the idea developed in the 1970s for use in fluidic valves [9] and later applied by Markland and co-workers [10] for switching gas turbine exit flows. The idea is to impart the tangential momentum by fixed vanes with control jets issuing from their trailing edges. The vanes form the swirl generator, located in the upstream part of the nozzle, Fig. 5. As shown in Fig. 6, the vanes are

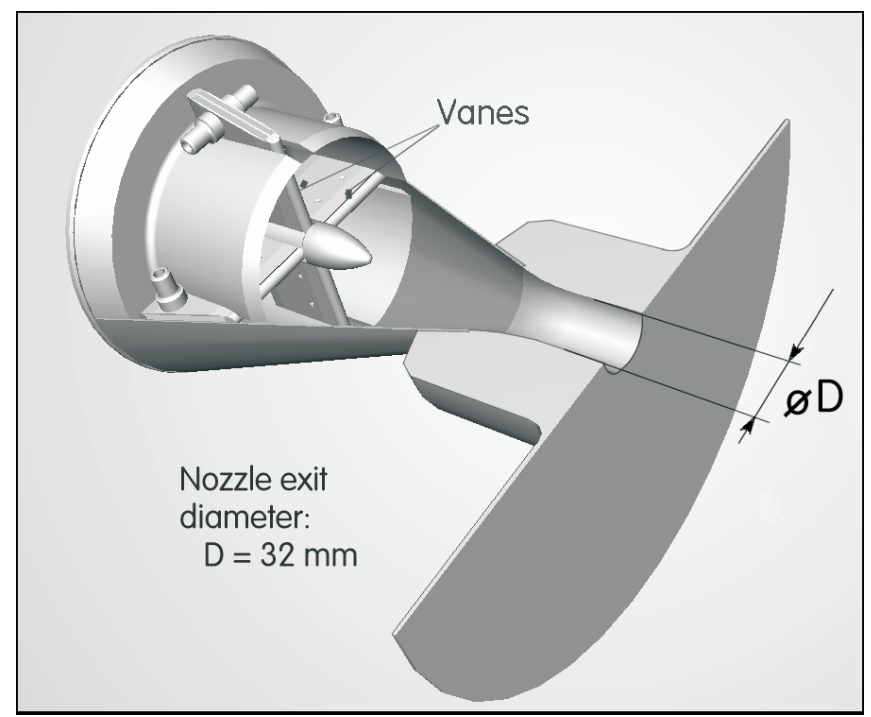

Fig. 5: The used nozzle with the original swirl generator based upon the supercirculation produced on fixed vanes by the Coanda effect.

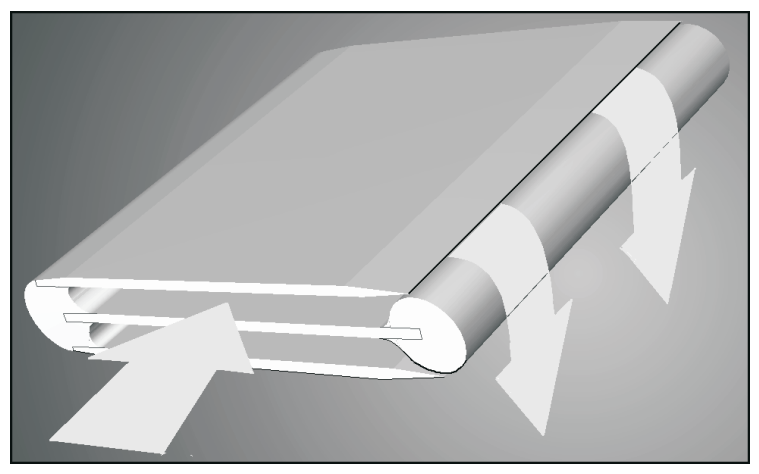

Fig. 6: The principle of supercirculation vanes as successfully developed at CTU in 1973 [9]. The ambichirality required in the present investigations called for two control flow exit slits (and two inlet cavities) in each vane.

hollow, made from parallel metal sheets and two round rods forming both the leading edge and the trailing edge. The thin jet issues from one of the slits between the outer sheets and the trailing edge rod (the other slit is used for generating the swirl of reverse chirality). The tangential momentum is imparted due to the circulation generated on the vane by the inclination of the jets (Fig. 6) caused by their attachment to the round trailing edges by the Coanda effect. A circulation around the vane is generated despite the geometric angle of attack of the vane being zero. The advantages of this novel swirl generator are:

a) As in all principles using vanes, by imparting the angular momentum over the whole radius the swirl generator produces a flat and regular resultant velocity profile.

b) The flat vane shape imparts no angular momentum without the control jet flow, generating a non-swirling jet.

c) Despite the flat vane shape, the magnification effect of the supercirculation makes it possible to impart angular momentum values at least an order of magnitude larger than with a vane at an adjusted attack angle (which is prone to separation at its leading edge if the attack angle is set too large).

d) The control effect is adjustable instantly, by changing the control air flow admitted into the hollow vanes (while mechanical adjustment of the vanes takes time) and because of absence of inertia of moving components the generated swirl may even be made time-dependent.

e) Thanks to the supercirculation effect, the control flow rate needed for a strong swirl is very small - an order of magnitude smaller (a magnification factor in excess of 20 was measured in [9]) than in the direct tangential inflow according to Fig. 4.

f) The swirl is reproducible and may be adjusted continuously - while mechanically adjusted vanes can be set reproducibly only if they are mutually linked by some mechanical linkages (which makes them expensive) or if they are provided with a set attack angle fixation mechanism. However in this case it is not possible to adjust them continuously, only to attack angles arranged in the mechanism.

g) The new Coanda jet vanes are simple and easy to make.

A virtual model of the swirl generator showing the four vanes and the small centre body is shown in Fig. 7. The vanes 


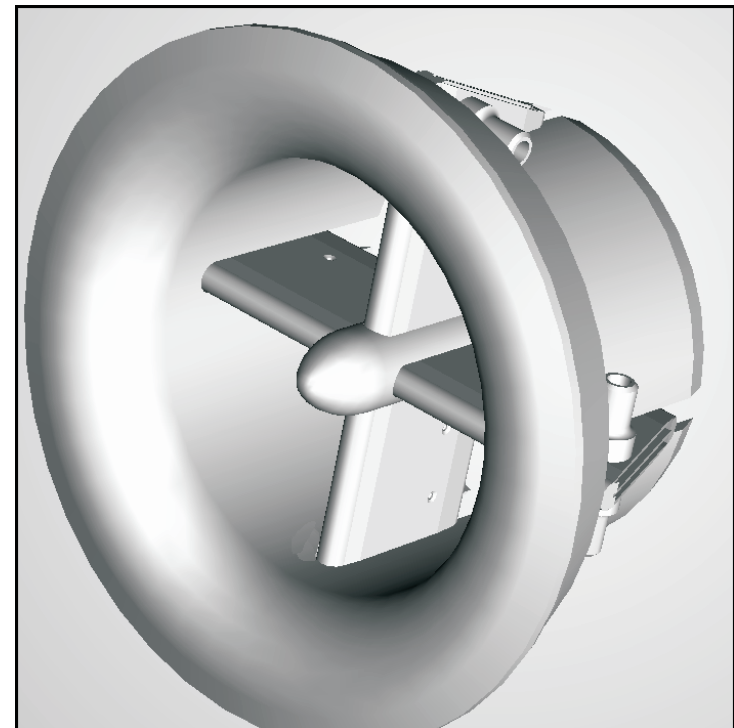

Fig. 7: Virtual computer model of the swirl generator from the upstream (inlet) side

are placed in the $112 \mathrm{~mm}$ dia upstream inlet part of the nozzle. The nozzle cross section decreases in the flow direction (Fig. 5) to the $D=32 \mathrm{~mm}$ exit, with a short constant-diameter channel. The vane chord is $52 \mathrm{~mm}$, thickness $9 \mathrm{~mm}$. The leading edge is of $r=4 \mathrm{~mm}$ radius, and the trailing edge is of smaller, $r=3.5 \mathrm{~mm}$ radius. On both sides of the trailing edge there is one $0.3 \mathrm{~mm}$ wide control flow exit slit. Fig. 8 presents a photograph of the nozzle and the generator as they were used in the first experiments (with air inlet connected for only one direction of generated swirl rotation).

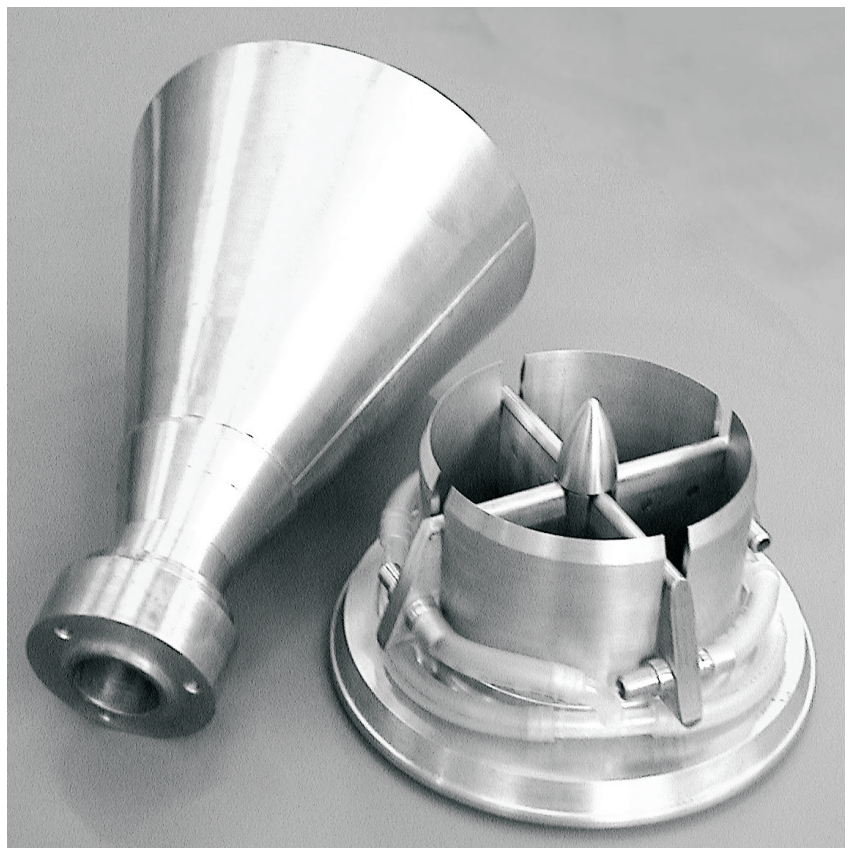

Fig. 8: Actual nozzle (left) and swirl generator (right) used in the experimental investigations

\section{Numerical model and preliminary tests}

Numerical flowfield computations were performed using a finite volume discretisation of the computation domain. Ini-

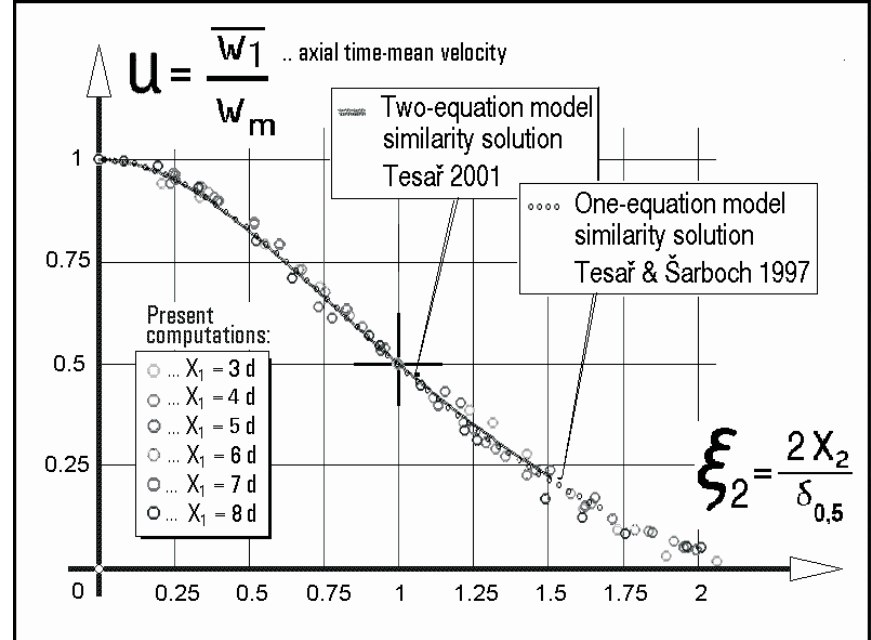

Fig. 9: With zero swirl the axial time-mean velocity profiles compare successfully with existing similarity solutions [16] and [17]

tially, the nominal symmetry of the flowfield was used to save some computation time by limiting the domain to only one quadrant (positive values of co-ordinates $X_{2}$ and $X_{3}$ ), with periodicity boundary conditions on the symmetry planes. The saving was, however, not large and there were some discrepancies caused by the enforced boundary conditions so that in the actual computation runs the domain was complete $3 \mathrm{D}$ volume. The downstream space where the investigated swirling jet is formed is cylindrical, $640 \mathrm{~mm}$ long, which is equivalent to 20 nozzle exit diameters. The diameter of this space is also $640 \mathrm{~mm}$, i.e. its transversal dimension is also equal to 20 nozzle exit diameters. While this may not suffice for a complete development of the turbulent structure of the generated jet (note that attaining the full equilibrium of turbulent fluctuation energy requires lengths larger than 60 nozzle diameters - cf. Fig.10), the size is definitely much larger than the region of interest in the experiments, and certainly suffices for evaluating the time-mean helicity (as is shown below, the region of substantial helicity magnitude does not extend to more than about 4 nozzle diameters - cf.

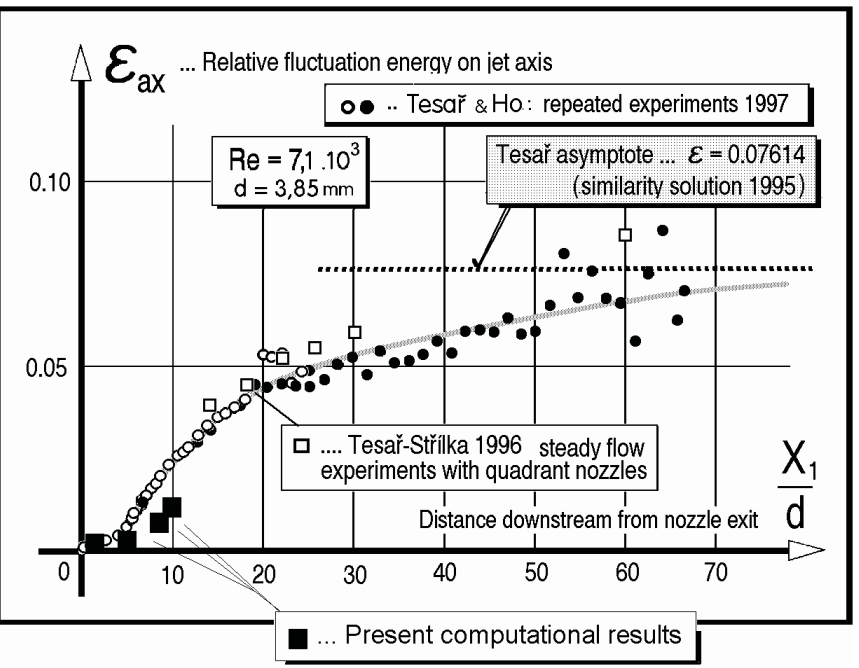

Fig. 10: Less satisfactory agreement with similarity solutions [17] for the turbulent fluctuation energy in the no-swirl case is explained by very long required development length, as found also in other investigations 
Fig. 18). The inlet into the nozzle in the experiment is from a large settling box. This is not modelled in its full extent, as experience has shown that with the character of the flow determined by the upstream boundary conditions it is sufficient to add to the domain just a small space uprstream from the nozzle. In the computations this upstream space was hemispherical, of $110 \mathrm{~mm}$ radius, as shown e.g. in Fig. 11.

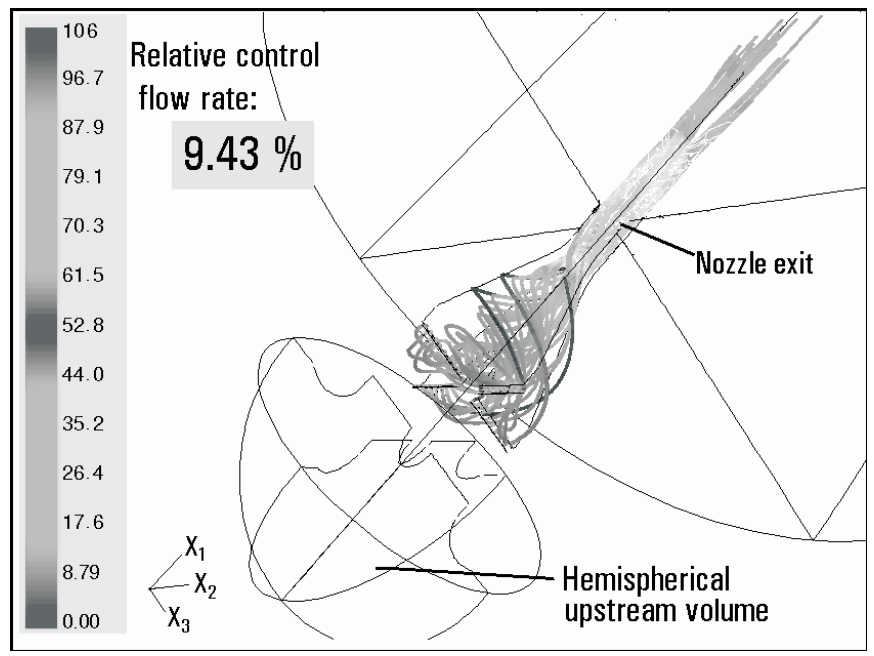

Fig. 11: Computed pathlines (colour coded by velocity magnitude) of the left-hand swirling flow downstream from the swirl generator at a medium magnitude of applied control

An unpleasant fact for computation is the presence of incongruent length scales. For efficient (and not excessively time-consuming) computations, the discretisation should lead to as small a number of grid elements as possible, all of them preferably not differing in size and having aspect ratios near to 1.0. In the investigated case there is, however, on the one hand the size of the control slits at the trailing edges of the swirl generator vanes as narrow as $0.3 \mathrm{~mm}$ - while there is the $640 \mathrm{~mm}$ long downstream volume. This means a more than 1:2000 ratio of the important scales. The elements of the discretisation grid cannot have such a span of sizes, and it was necessary to use a larger number of small elements to cover the large space. After the grid adaption, performed by refinement in regions of large velocity magnitude gradient during the initial runs, the final unstructured tetrahedral discretisation contained as many as 921167 cells.

The computations were run using the two-equation turbulence model with the with standard set of model constants and the RNG approach for modification of the model in regions of low turbulence Reynolds numbers. Prior to the actual swirling jet computations, verification runs were performed with zero control flows from the control slits in the vanes. The intention was to confront the used computational procedure with known results. In particular, the computed profiles of time-mean velocities and turbulence parameters were compared with the similarity solution of the axisymmetric turbulent jet using the one-equation model of turbulence by the present author and his student J. Šarboch [16], and the later analogous similarity solution using the two-equation model of turbulence described in [17]. The computed axial velocity profiles were used to evaluate the convention diameter $\delta_{0.5}$, defined as the transversal distance between the two locations in which the axial time-mean velocity equals $0.5 w_{\mathrm{m}}$, where $w_{\mathrm{m}}$ is the maximum axial velocity at the same downstream distance $X_{1}$. The profiles were converted into similarity co-ordinates, as shown in Fig. 9, with local time-mean axial velocity related to the velocity maximum in the particular profile and the transverse distance from the jet axis related to the convention jet diameter. As seen in Fig. 9, all profiles were indeed found to be similar - which, in fact, is somewhat surprising since this similarity was found at downstream distances from the nozzle less than the usually encountered development lengths. Fig. 9 also documents excellent agreement of the computed data points with the available similarity solutions from [16] and [17].

Similar comparisons were also made for the computed specific values of the fluctuation energy (per unit mass - as seen from the dimension $\mathrm{m}^{2} / \mathrm{s}^{2}$ ) nondimensionalised by relating to the square of the maximum velocity $w_{\mathrm{m}}$. The computed values were much lower than the equilibrium values assumed in the similarity solutions. This, however, is not surprising in view of the fact that the jet leaves the nozzle with a very small, almost negligible level of turbulent fluctuations. The fluctuations are produced in the shear layers of the jet and this leads to a considerable downstream distance before turbulence assumes the fully developed state. This downstream distance is much longer than generally believed - as demonstrated in the present author's earlier experimental measurements (with his student T. Střílka - [17]) presented in Fig. 9, where a length as large as equal to 60 nozzle exit diameters was required for complete development. The present results are quite in line with the general character of the experimental data in Fig. 9, where the values of the dimensionless fluctuation energy on the jet axis are plotted as a function of the relative downstream distance.

The most important conclusion from these validation computations is the substantial difference between the advection dominated case of the velocity field and the production dominated specific energy field. The profiles of the former exhibit a local maximum on the jet axis and values decreasing monotonously with the streamwise distance. On the other hand, the latter exhibit an off-axis maximum and a gradual streamwise growth in the locations of present interest immediately downstream from the nozzle exit.

\section{Helicity computation results}

The investigations being rather time-consuming, only five cases of swirling jets (listed in Table I ) have been evaluated so far. They, however, suffice for formulation of some useful more general results. All flows investigated so far have concentrated on quite high Reynolds number regimes. The four cases A, B, C, and D share the same flow rate magnitude supplied into the axial inlet and differ in the magnitude of the control flow rate admitted into the vane exit slits (distributed equally into all four slits). The four control flow rate magnitudes in the tests increase roughly in a geometric series. In the cases $\mathrm{C}$ and $\mathrm{D}$, in fact, all flow rates were the same and the only difference was the control flow issued from the other set of four slits, producing the reverse rotation sense (direction). The last two cases D and E, on the contrary, share the same magnitude of the control flow rate and differ in the magnitude of the axial flow rate. 
Table I: List of investigated flows. The otherwise identical cases C and $\mathrm{D}$ differ in left-handed and right-handed direction of swirl rotation. Characteristic swirl angle $\alpha$ is evaluated from the ratio of tangential and axial velocity maxima in the nozzle exit.

\section{COMPUTED SWIRLING JET CASES}

\begin{tabular}{|c|c|c|c|c|}
\hline & $\begin{array}{l}\text { Relative control } \\
\text { flow rate }\end{array}$ & chirality & $\mathrm{Re}$ & $\begin{array}{c}\alpha \\
{[\mathrm{deg}]}\end{array}$ \\
\hline A & $4.42 \%$ & $\mathrm{R}$ & $17810^{3}$ & 4.9 \\
\hline B & $6.45 \%$ & $\mathrm{R}$ & $18210^{3}$ & 8.9 \\
\hline C & $9.43 \%$ & L & $18910^{3}$ & -12.5 \\
\hline D & $9.43 \%$ & $\mathrm{R}$ & $18910^{3}$ & 12.5 \\
\hline E & $12.6 \%$ & $\mathrm{R}$ & $14810^{3}$ & 14.6 \\
\hline
\end{tabular}

\begin{tabular}{|lc|}
\hline $1.06 \mathrm{e}+02$ & \\
$9.67 \mathrm{e}+01$ & Relative \\
$8.79 \mathrm{e}+01$ & control flow rate \\
$7.91 \mathrm{e}+01$ & $9.43 \%$ \\
$7.03 \mathrm{e}+01$ & \\
$6.15 \mathrm{e}+01$ & \\
$5.28 \mathrm{e}+01$ & \\
$4.40 \mathrm{e}+01$ & \\
$3.52 \mathrm{e}+01$ & Path Lines Colored \\
$2.64 \mathrm{e}+01$ & by Velocity Magnitude $(\mathrm{m} / \mathrm{s})$ \\
$1.76 \mathrm{e}+01$ & $\mathrm{X}_{3}$ \\
$8.79 \mathrm{e}+00$ & $\mathrm{X}_{2}$ \\
$0.00 \mathrm{e}+00$ & $\mathrm{X}_{1}$ \\
\hline
\end{tabular}

Fig. 12: Computed pathlines (colour coded) released from a tangentially located line so that they approach one of the vanes in a tangential plane perpendicular to the vane span.

As the first observation, the computed pathlines in Fig. 11 and Fig. 12 as well as the experience gained with the laboratory rig indicate very satisfactory operation of the novel swirl generator. This was already seen from the very small values of the relative control flow rates sufficient for obtaining quite intensive swirl intensity. In spite of the rather small radius (only $r=3.5 \mathrm{~mm}$ ) of the vane trailing edges, the jet sheets issuing from the control slots safely attach to them and generate the required circulation about the vanes. The quality of the flow issuing from the nozzle is completely satisfactory. The example of evaluated tangential velocity profiles in Fig. 13 shows that the generated jet rotation has a solid body character (velocity increasing in proportion to radial distance) - decaying quite rapidly, however, as soon as the jet leaves the nozzle.

As for the evaluated helicity, because of the rather complicated evaluation procedure and also because of the differentiation operation involved in computing the vorticity, the scatter of the data is quite large, even though the velocities have converged to smooth spatial distributions. There is also a remarkable difference in the size of the velocity and helicity flowfields. The helicity dissipates and diffuses away very fast

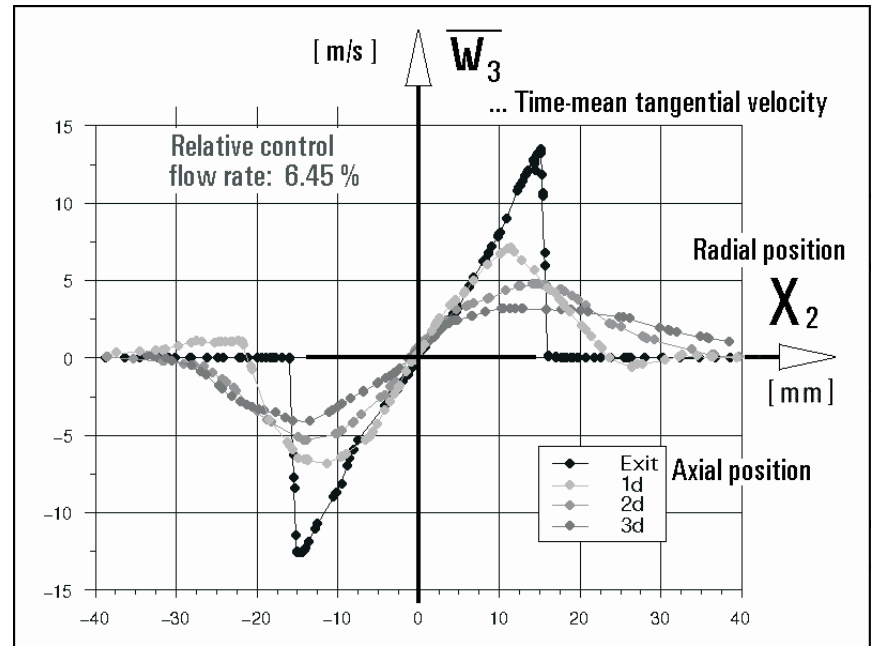

Fig. 13: Typical profiles of tangential velocity and their changes with axial distance downstream from the nozzle exit

once the jet leaves the nozzle, while the velocity field exhibits significantly nonzero values in a large part of the available downstream volume. A decrease of helicity magnitude to only $5 \%$ (i.e. to within the likely error of the results) of the initial nozzle exit value was found to take place at a distance shorter than 4 exit diameters.

\subsection{Two helicity generating mechanisms}

The first among the interesting results which do not seem to have been discussed in the available literature is the discovered existence of two different regions with significantly increased helicity: one of them is the solid-body-rotation core, and the other is the boundary layer on the inner wall of the nozzle. The two regions are discernible downstream from the nozzle in Fig. 14. The regions may even exhibit reverse chirality. If a positive helicity (right-handed) is generated by the swirle generatorr vanes in the core, as shown in Fig. 15, then the helicity in the near-wall layer is negative - Fig. 16.

Quite surprisingly, no doubt due to the pseudo-scalar nature of helicity, reversing the sense (direction) of the generated rotation did not lead to a simple change in the sign of the

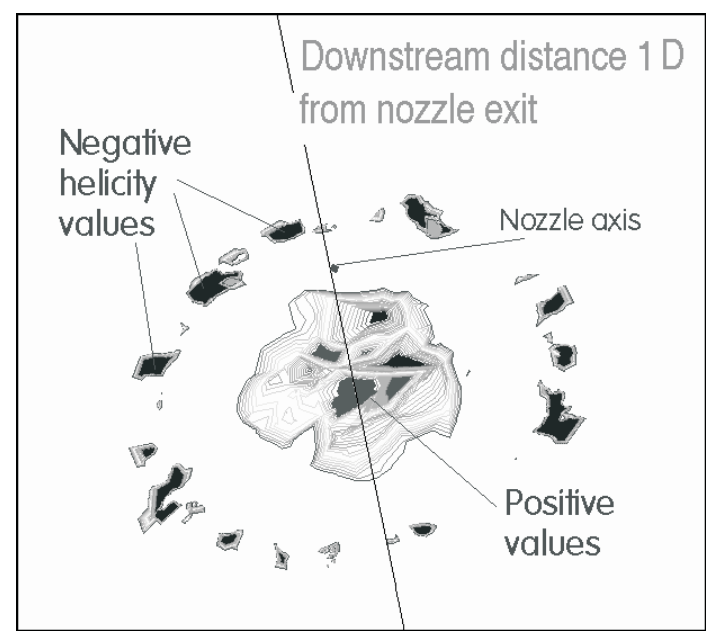

Fig. 14: Typical contours of positive and negative helicity in the perpendicular plane downstream from the nozzle exit evaluated for flow with positive (i.e. right-handed) swirl chirality 


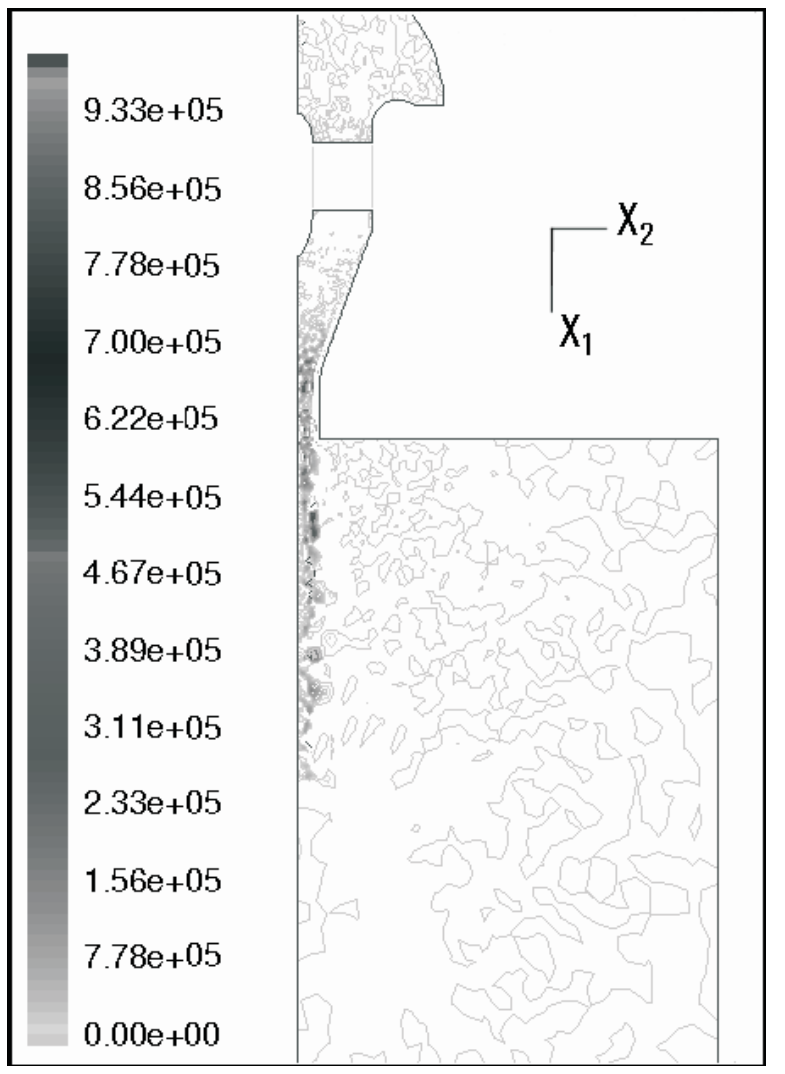

Fig. 15: Typical positive helicity contours evaluated in the meridian plane for a jet with positive swirl chirality - case D, with medium applied relative control flow rate $9.43 \%$

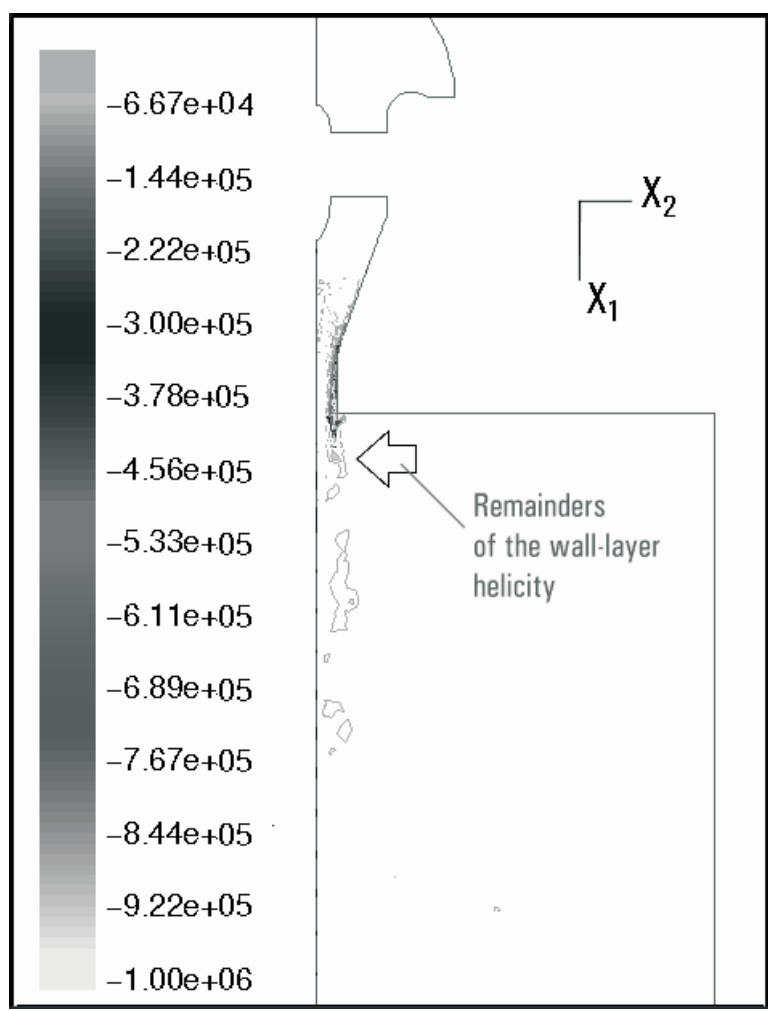

Fig. 16: Negative helicity contours evaluated in the same meridian plane for the same jet with positive swirl chirality as in Fig.15 (the negative valued helicity is generated in the boundary layer on the nozzle wall)

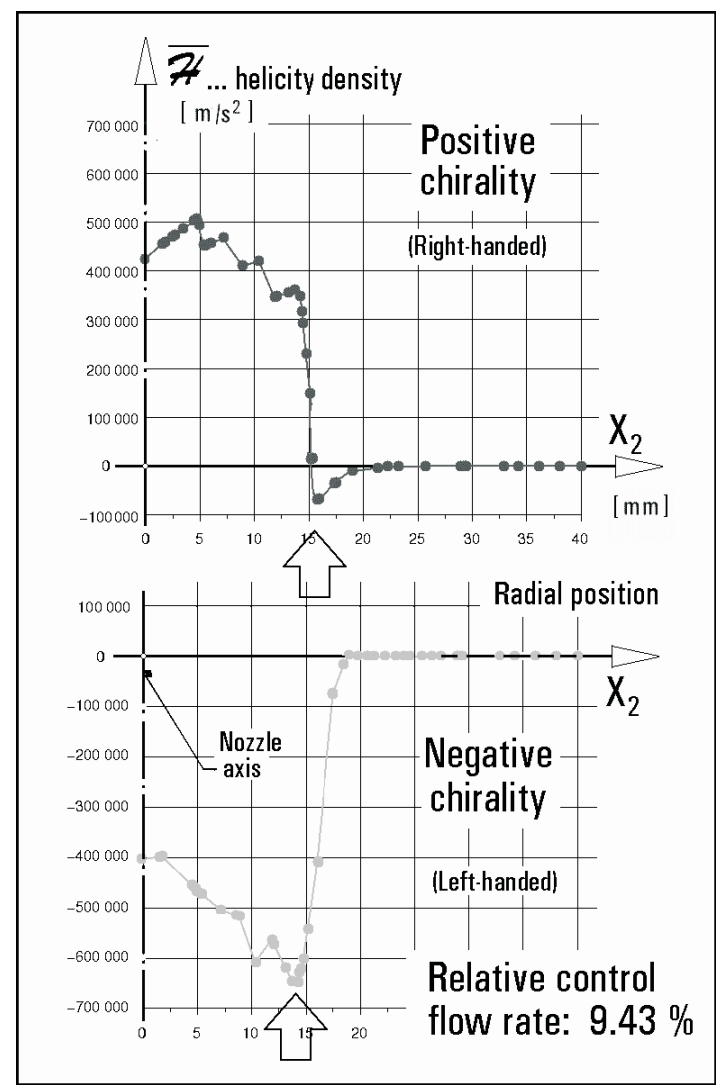

Fig. 17: The consequence of the pseudo-scalar character of helicity: reversal of chirality of the imparted swirl does not lead to a simple change in the sign of the helicity. Half-profiles of helicity evaluated immediately downstream from the nozzle exit indicate invariance of the sign of the helicity in the nozzle wall boundary layer.

results. The sign of the helicity generated in the wall layer tended to be the same (negative), so that the resultant helicity in the nozzle exit is subtracted in the positive swirl chirality flow case C (as shown in the top part of Fig. 17) and added to the negative swirl chirality in the flow case D (in the bottom part of Fig. 17).

Nevertheless, the near-wall helicity decays considerably faster, and while it remains detectable (Fig. 16) - note also the

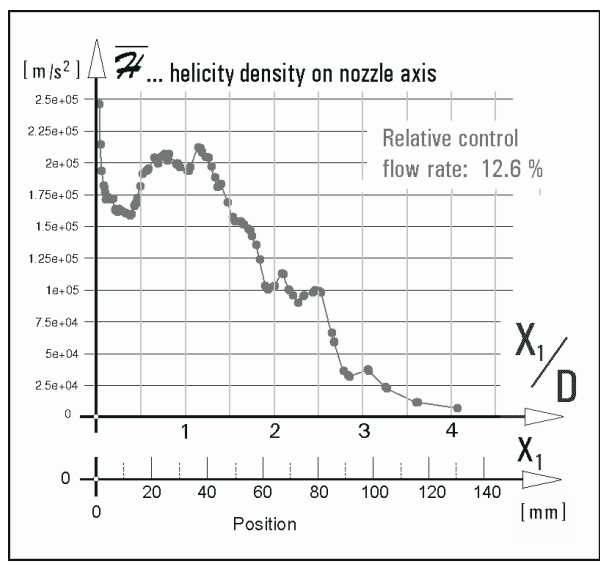

Fig. 18: Helicity distribution along the jet axis shows the remarkably fast decrease: values become less than the typical uncertainty beyond the downstream distance from the nozzle exit equal to 3-4 exit diameters 
vestigial negative values in the example profiles in Fig. 21 at the distance equal to one nozzle exit diameter - it ceases to be an important factor in the velocity profiles evaluated further downstream (though it may be responsible for the lower values indicated by the arrow in Fig. 23).

\subsection{Similarity of helicity profiles in the jet}

The profiles of the time-mean helicity density were evaluated from the individual components of the velocity and vorticity vectors by computing the time-mean variant of the expression Eq.(1)

$$
\overline{\mathcal{H}}=\bar{w}_{1} \bar{\omega}_{1}+\bar{w}_{2} \bar{\omega}_{2}+\bar{w}_{3} \bar{\omega}_{3} \ldots
$$

As an example, Fig. 19 presents the values evaluated at a distance of 2 nozzle diameters from the exit for the case $\mathrm{E}$, showing the profiles of the six individual quantities from eq.(2) as well as the three products to be summed. The substantial difference in the vertical scales may be noted: the resultant helicity density profile presented in Fig. 20 is clearly dominated by the axial component with just a secondary effect of the tangential values.

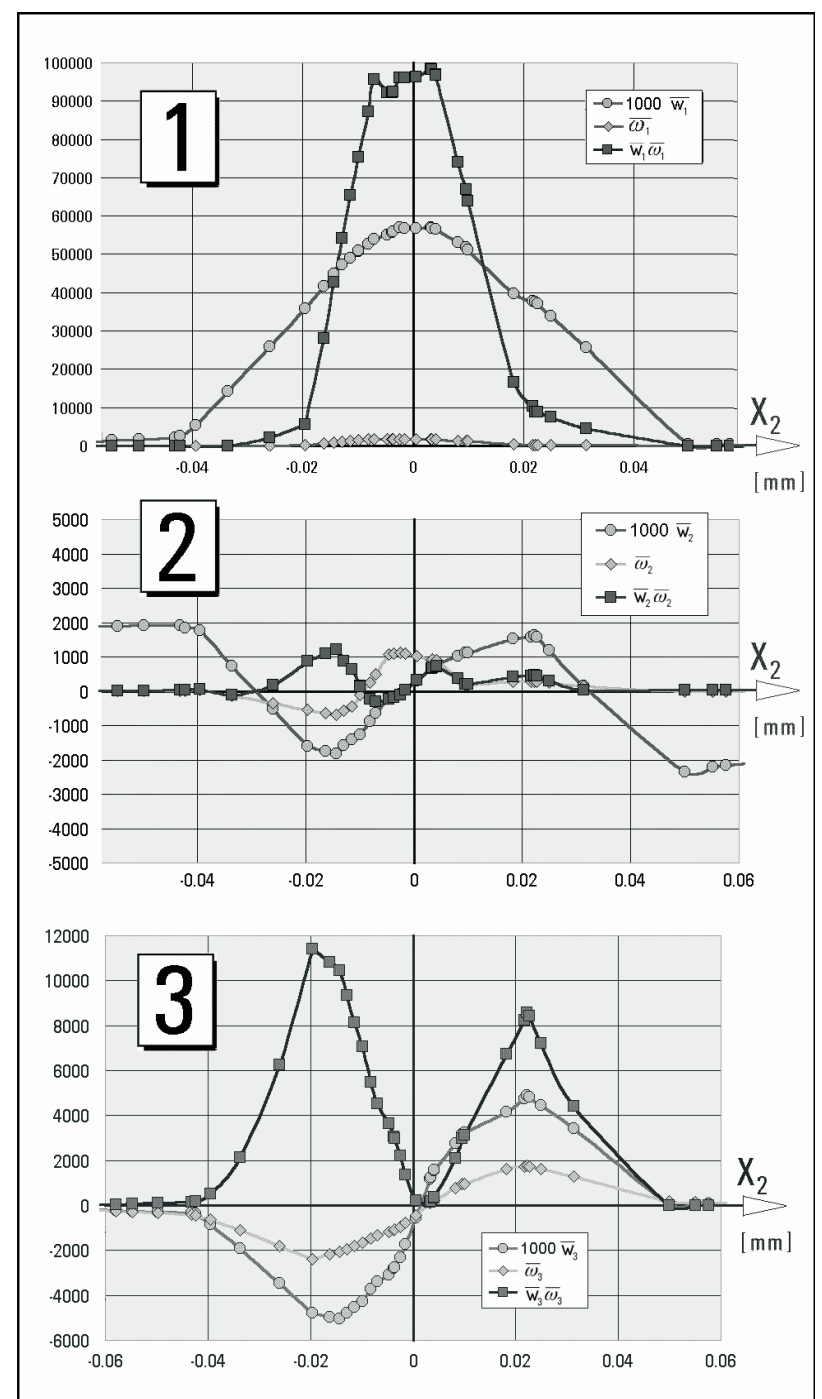

Fig. 19: Evaluation of helicity from the three velocity components and the three components of the vorticity vector. 1-axial components, 2- radial components, 3- tangential components (note the different vertical scales). Computed for $12.6 \%$ relative control flow rate at $\mathrm{X}_{1}=2 D$.

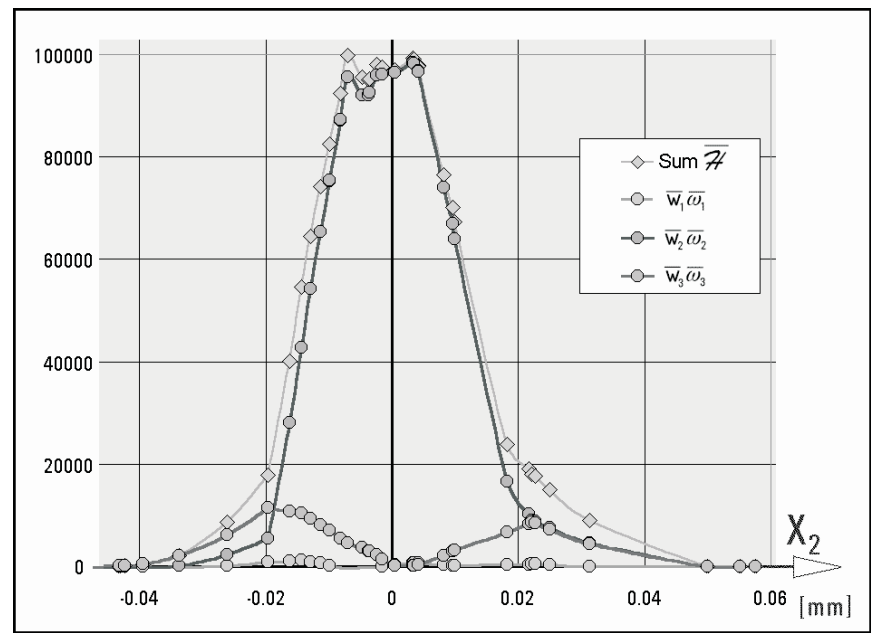

Fig. 20: Profiles of the three velocity $\times$ vorticity products from the example in Fig. 19 and the helicity profile as the result of their sum

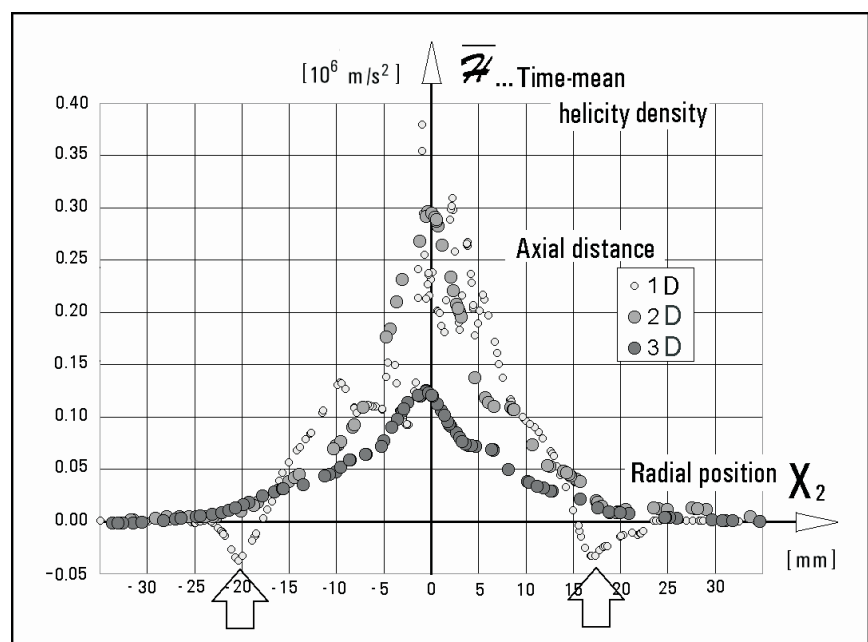

Fig. 21: Typical helicity profiles evaluated at several small distances downstream from the nozzle for a small, $6.45 \%$ relative control flow rate

In the computed helicity profiles, e.g. those in Fig. 21, it is easy to evaluate the maximum values $\overline{\mathcal{H}}_{\text {max }}$ - usually very

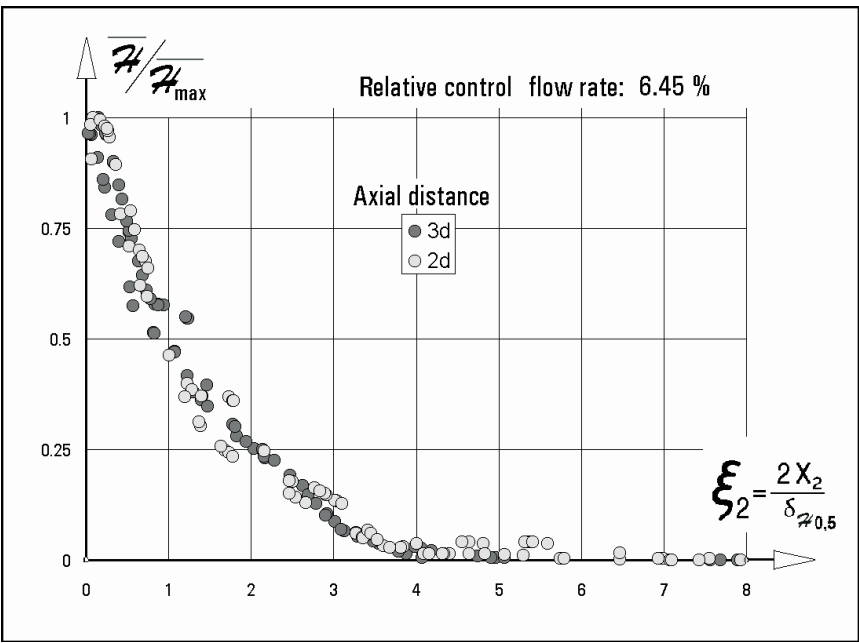

Fig. 22: Similarity the of two helicity profiles from Fig. 21 when plotted in relative co-ordinates 


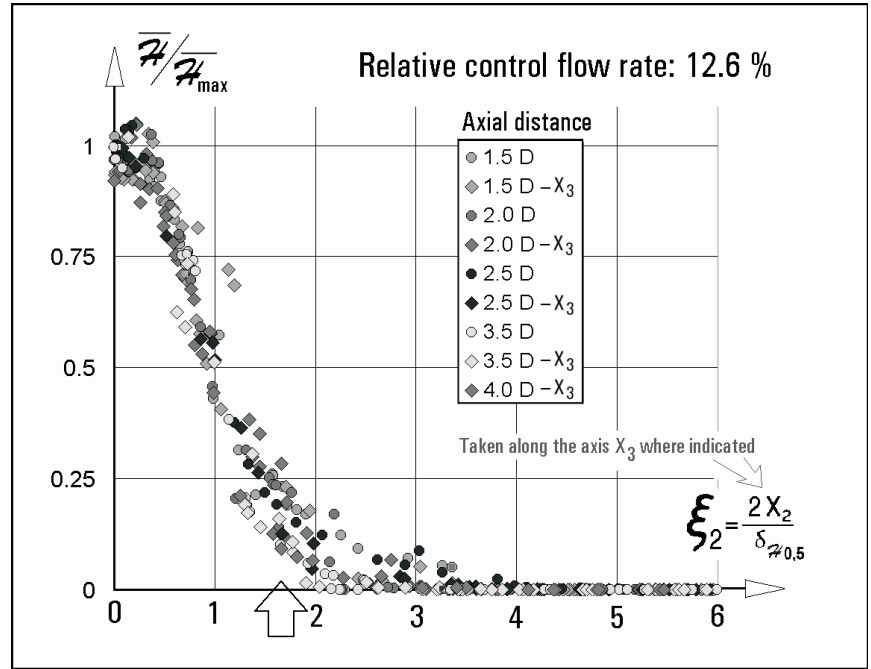

Fig. 23: Similarity of helicity profiles evaluated for the highest investigated control flow rate. The wide arrow (cf. Fig. 17 and Fig. 21) indicates low values, which may be a remnant of the negative wall-layer helicity.

near to the time-mean helicity density on the jet axis. They may then be used to nondimensionalise the computed and experimental values of helicity. It is then also easy to find the locations in which the local time-mean helicity density magnitude equals a pre-determined percentage of the maximum. In the present case, the author has determined the radial locations in which the interpolated helicity value is equal to $1 / 2 \overline{\mathcal{H}}_{\text {max }}$ at the same downstream distance $X_{1}$. These locations define the convention diameter $\delta_{\mathcal{H}_{0.5}}$ used to non-dimensionalise the horizontal co-ordinate. The profiles of time-mean helicity density exhibit a remarkable mutual similarity and in the transformed co-ordinates, as seen in the examples Fig. 22 to Fig. 24, the resultant nondimensionalised profiles collapse to almost a single curve - with some scatter understandable considering the rather involved evaluation procedure involving the spatial derivatives.

Also the transverse size of the helicity region, characterised by the diameter $\delta_{\mathcal{H}_{0.5}}$, exhibits a considerable scatter. Despite this, Fig. 25 tends to suggest that the size increases in the downstream direction in a linear manner, in agreement

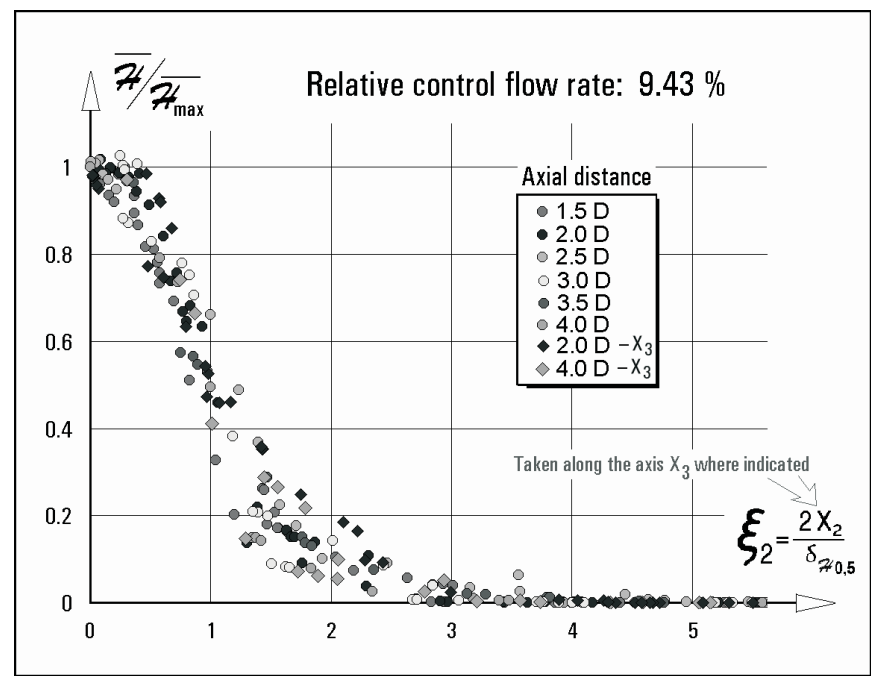

Fig. 24: Similarity of helicity profiles evaluated from the medium control flow rate computation results

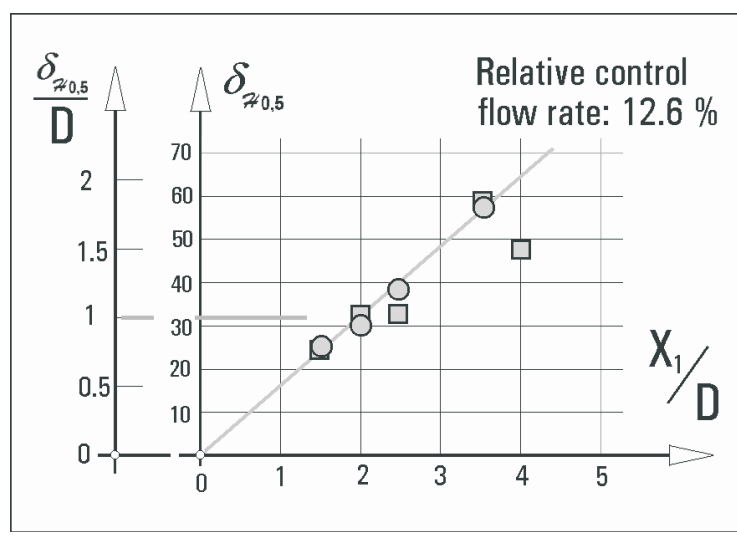

Fig. 25: Axial growth rate of the convention diameter of the helicity region in the investigated swirling jet evaluated from the data used in plotting the profiles in Fig. 23

with the idea of passive transport by turbulent vortical motions. This idea may form a basis for future formulations of helicity transport equations. Fig. 26 shows an example of an early result in this direction, based upon the idea of a constant helicity diffusion coefficient in a constant velocity field. The latter simplifying assumption stems from the observation that the profiles are mostly located in the constant-velocity "potential" core of the jet.

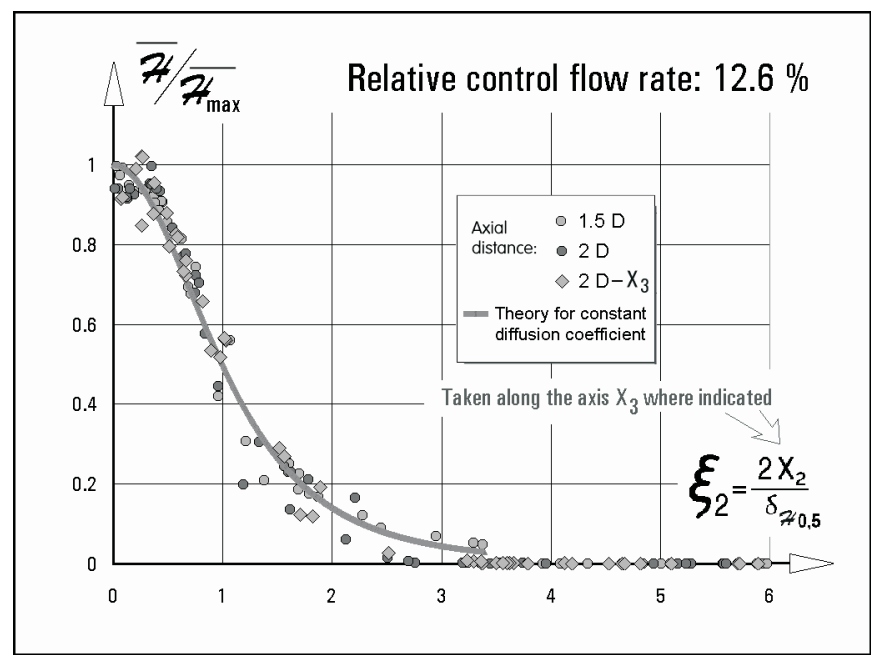

Fig. 26: Comparison of typical evaluated helicity profiles in relative co-ordinates and a theoretical similarity solution for a constant value of the turbulent gradient transport

\section{Conclusions}

The concept of helicity used for investigations of turbulent flowfield, after a period of mixed previous results, is currently gaining popularity. Spatial distributions of the time-mean component of helicity density discussed in this paper are the starting point. Unfortunately, very little information has been available. The presented results of continuing investigations, obtained using parallel experimental and computational approaches, provide some information about the case of a turbulent swirling jet. The swirl was imparted by an novel method, using an unusual swirl generator design based upon the author's earlier idea of producing flow deflection by using Coanda-effect generated supercirculation on fixed vanes. The design proved to be very useful, bringing several marked 
advantages. The investigations of the generated time-mean helicity density in the swirling jet indicate that the region forming donwstream from the nozzle is of limited extent, the time-mean helicity apparently becoming converted into the stochastic helicity component. Very important finding of this study is the fact that the evaluated profiles of time-mean helicity density exhibit remarkable similarity.

\section{Acknowledgments}

This research has been supported by the grant "Novel merging swirl burner design controlled by helical mixing" provided by EPSRC - Engineering and Physical Sciences Research Council, U.K. - to W. B. J. Zimmerman and N. Russel. The experimental rig was made by Mr. Christopher Turner. The measurements in the swirling jets by Particle Image Velocimetry, performed under the author's supervision by Miss Gavita Regunath, though her results were not used in this paper, provided a useful insight into swirling jet flows.

\section{References}

[1] Moffatt, H. K.: "Degree of Knottedness of Tangled Vortex Lines.” J. Fluid. Mech., Vol. 36 (1969), p. 117-129.

[2] Moffatt, H. K., Tsinober, A.: "Helicity in Laminar and Turbulent Flow.” Ann. Rev. Fluid Mech., Vol. 24 (1992), p. 281-312.

[3] Lord Kelvin: "On Vortex Motion.” Trans Roy. Society Edin., 25, 1869, p. 217-260.

[4] Moreau, J. -J.: "Constants d'un ilot tourbillionaire en fluide parfait barotrope.” C. R. Acad. Sci. Paris Vol. 252 (1961), p. 2810-2812.

[5] Ditlevsen, P. D., Giuiani. P.: “A Note on Dissipation in Helical Turbulence.”, Physics of Fluids, November 2001.

[6] Biferale, L., Pierotti, D., Toschi, F.: "Helicity Transfer in Turbulent Models.” Physical Review E, Vol. 57 (1998), Nr. 3, March 1998.

[7] Tesař, V.: "The Problem of Round Jet Spreading Rate Computed by Two-Equation Turbulence Model." Proc. of Conference "Engineering Mechanics '97", Svratka, Czech Republic, May 1997, p. 181.

[8] Suzuki, Y., Nagano, Y.: "Modification of Turbulent Helical / Non-Helical Flows with Small-Scale Energy Input." Phys. Fluids, Vol. 11, No. 11, p. 3499-3511.

[9] Tesař, V.: „Fluidické proudové výkonové prvky se supercirkulační intensifikací hybnostní interakce proudů.“ (Power Fluidic Jet-Type Elements with Supercirculation Intensification of Jet Momentum Interaction, in Czech), Acta Polytechnica - CTU in Prague, Czech Republic 1973.
[10] Markland, E., Tsevdos, N.: "The Jet Flap Applied to Flow Control." Proc. of $2^{\text {nd }}$ International Symposium on Fluid Control, Measurement, Mechanics, and Visualisation. FLUCOME '88, Sheffield, 1988.

[11] Khomenko, G. A., Zimmerman, W. B.: "Large Scale Structure Evolution and Mixing Due to Small Scale Helical Forcing in a Compressible Viscous Fluid.” In: Mixing in Geophysical Flows, Eds. J. M. Redondo and O. Metais, UPC Barcelona Press, 1994, p. 233-248.

[12] Zimmerman, W. B.: "Fluctuations in Concentration Due to Convection by a Helical Flow in a Conducting Fluid." Phys. of Fluids, Vol. 8 (1996), No. 6, p. 1631-1641.

[13] Zimmerman, W. B.: "Fluctuations in Passive Tracers Due to Mixing by Coherent Structures in Isotropic, Homogeneous, Helical Turbulence." Inst. Chem. Eng. Symposium Series 140, 1996, p. 213-224.

[14] Tesař, V.: "Two-Equation Turbulence Model Solution of the Plane Turbulent Jet." Acta Polytechnica, Vol. 35 (1995), No. 2, p. 19-42, Prague, ISSN 1210-2709.

[15] Tesař, V.: "The Solution of the Plane Turbulent Jet." Acta Polytechnica, Vol. 36 (1996), No. 3, p. 15-41, Prague, ISSN 1210-2709.

[16] Tesař, V., Šarboch, J.: "Similarity Solution of the Axisymmetric Turbulent Jet Using the One-Equation Model of Turbulence.” Acta Polytechnica, Vol. 37 (1997), No. 3, p. 5-34, Prague, ISSN 1210-2709.

[17] Tesař, V.: “Two-Equation Turbulence Model Similarity Solution of the Axisymmetric Fluid Jet." Acta Polytechnica, Vol. 41 (2001), No. 2, p. 26-41, Prague, ISSN 1210-2709.

[18] Tollmien, W.: "Berechnung turbulenter Ausbreitungsvorgänge." Zeitschrift für Angewandte Mathematik und Mechanik, Vol. 6 (1926), p. 468, Berlin, Germany.

[19] Tesař, V.: "Similarity Solutions of Basic Turbulent Shear Flows with One- and Two-Equation Models of Turbulence." Zeitschrift für Angewandte Mathematik und Mechanik, Vol. 77 (1997), p. 333, Berlin, Germany.

Prof. Ing. Václav Tesař, CSc.

e-mail: v.tesar@sheffield.ac.uk

University of Sheffield

Mappin Street S1 3JD

Sheffield, UK

AV ČR

Dolejškova 5

18200 Praha 8, Czech Republic 